\title{
Neuropilin1, a novel independent prognostic factor and therapeutic target in triple-negative breast cancer
}

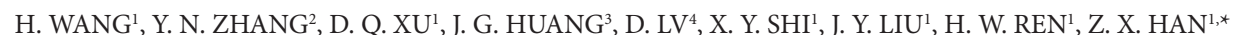

${ }^{1}$ Department of Oncology, The Affiliated Hospital of Xuzhou Medical University, Xuzhou Medical University, Xuzhou, China; ${ }^{2}$ Department of Oncology, Xuzhou No. 1 People’s Hospital, Xuzhou, China; ${ }^{3}$ Department of Oncology, Peixian People's Hospital, Xuzhou, China; ${ }^{4}$ Department of General Surgery, The Affiliated Hospital of Xuzhou Medical University, Xuzhou Medical University, Xuzhou, China

${ }^{\star}$ Correspondence: cnhzxyq@163.com

Received November 27, 2019 / Accepted March 4, 2020

\begin{abstract}
Triple-negative breast cancer (TNBC) is the most aggressive breast cancer, and thus, has limited treatment options. Neuropilin1 (NRP1) is a multi-functional transmembrane protein that interacts with a number of signaling receptors and plays an important role in cancer progression. Previous studies demonstrated that the expression of NRP1 is activated and promotes the progression of breast cancer particularly in TNBC compared to other molecular subtypes; however, whether or not the level of NRP1 expression is related to the progression of TNBC warrants further study. In the current study, we determined the expression and function of NRP1 and evaluated the clinical significance of NRP1 in patients with TNBC. In addition, we determined whether or not an NRP1 antagonist potentiates the inhibitory effects of paclitaxel (PTX) in patients with TNBC. In our clinical study, NRP1 had higher expression in TNBC tissues than non-TNBC tissues at the same stage, and NRP1 was an independent prognostic factor. Specifically, the high expression of NRP1 was associated with shorter survival in TNBC patients. In addition, TNBC cells treated with NRP1 antagonist significantly potentiated the effect of PTX on cell proliferation, cell migration, and apoptosis. Our findings suggest that NRP1 expression can act as an independent prognostic factor for TNBC patients, and the combination of PTX and an NRP1 antagonist may be an effective treatment regimen for TNBC.
\end{abstract}

Key words: triple-negative breast cancer, chemotherapy, neuropilin1, paclitaxel

Breast cancer is the most common cancer and the second most common cause of cancer-related deaths in women [1]. There are four main female breast cancer subtypes (luminal A, luminal B, HER2-positive, and triple-negative [TNBC]). TNBC, a special type of basal-like breast cancer, comprises $15-20 \%$ of all breast cancers, first appeared in the literature in 2005 [2] and is defined by the absence of estrogen receptor (ER), progesterone receptor (PR), and Her2 expression [3]. TNBC is characterized by a highly aggressive clinical course, including a higher metastasis rate to the central nervous system and internal organs [4]. Although newer regimens are gaining acceptance, no targeted therapy has been approved to treat TNBC $[5,6]$. Traditional chemotherapy drugs, such as paclitaxel (PTX), often fail to completely eradicate the tumor, resulting in recurrences [7].

Neuropilin1 (NRP1) was first discovered in nerve axons by Fujisawa's group in 1995 [8] and was initially described as a co-receptor for secreted semaphorins and vascular endothe- lial growth factors (VEGFs). Clinical evidence suggested that the upregulation of NRP1 occurs in a variety of malignancies, such as pancreatic cancer, lung cancer, ovarian cancer, and gastrointestinal tumors, and high expression of NRP1 promotes tumor proliferation, invasion, and metastasis [9-11].

Recent research has suggested that NRP1 is highly expressed in breast cancer [12]. Our previous study demonstrated that NRP1 participates in the progression of breast cancer [13]. RNA interference (RNAi)-mediated NRP1 silencing suppresses proliferation, promotes apoptosis, and enhances sensitivity to chemotherapeutic agents [14]; however, little is known with respect to the biological functions NRP1 may have in TNBC. In this study, we determined the expression and function of NRP1 and evaluated the clinical significance in TNBC by immunohistochemistry. To extend the therapeutic regimen to TNBC, we determined the combined therapeutic effect of an NRP1 antagonist with PTX. 


\section{Patients and methods}

Patients and specimens. The protocol was approved by the Ethics Committee of the Affiliated Hospital of Xuzhou Medical University (Xuzhou, China) in accordance with the guidelines of the Declaration of Helsinki. Paraffin-embedded specimens from 69 TNBC patients who underwent surgery at the Affiliated Hospital of Xuzhou Medical University between January 2008 and January 2018, together with 60 non-TNBC patients, were reviewed and selected from the archives of the Department of Pathology and Research Laboratory (Affiliated Hospital of Xuzhou Medical University, Xuzhou, China). As recommended by the World Health Organization (WHO) criteria, two pathologists independently confirmed the histopathologic findings and the diagnosis of each case. Neo-adjuvant chemotherapy, endocrine therapy, or radiotherapy was not administered before surgery. Specimens from patients who underwent breast cancer surgery were divided into post-operative pathologic stages according to the size of the primary tumor, regional lymph node metastasis, and whether or not there was distant metastasis. The degree of differentiation (high, medium, and low) represented the difference between tumor cells and normal tissue cells. All of the patients were female, and the mean age of the 129 breast cancer patients was 56.5 years (range, 31-82 years). The endpoint of the research was overall survival (OS). The OS was the period from the date of surgery until death or to the last follow-up evaluation. All 129 patients were regularly followed at 2-3 month intervals for 36 months.

Immunohistochemistry (IHC). All 129 surgically excised tumors were fixed in $10 \%$ neutral formalin, embedded in paraffin, and cut into $4 \mu \mathrm{m}$ sections. IHC for NRP1 was performed using standard techniques and the S-P method. Positive cancer tissue sections were used as positive controls, and phosphate-buffered saline (PBS) solution instead of the primary antibody served as a negative control. The S-P kit instructions were strictly followed. Specifically, after dewaxing, dehydration, and antigen retrieval, $3 \% \mathrm{H}_{2} \mathrm{O}_{2}$ was applied to eliminate endogenous peroxidase activity for $10 \mathrm{~min}$ at $37^{\circ} \mathrm{C}$, and normal goat serum was used to block non-specific reactivity for $10 \mathrm{~min}$ at $37^{\circ} \mathrm{C}$. Sections were then incubated overnight at $4^{\circ} \mathrm{C}$ with polyclonal rabbit anti-NRP1 antibody at a 1:100 dilution (EPR3113; Abcam, USA). This concentration was the optimal ratio based on early preliminary experiments. Sections were incubated sequentially with biotinylated goat anti-rabbit immunoglobulin and peroxidase-conjugated streptavidin (Zhongshan, China), then 3'-diaminobenzidine tetrahydrochloride (DAB) was applied for the immunostaining reaction, followed by Meyer hematoxylin counterstaining.

Evaluation of staining. We mainly evaluated the staining of breast cancer cells. The final immunohistochemical score by IHC score was based on a double scoring system, which was generated from the proportion of positively stained cells combined with staining intensity, thus, producing a total range of $0-6$. The staining area proportion (A) was scored as follows: 0 , absent staining of cells in any microscopic field; $1,1-25 \% ; 2,26-50 \%$; and $3,>50 \%$. The staining intensity score (B) was as follows: 0 (-, no staining); 1 (+, definite, but weak staining); 2 (++, moderate staining); and $3(+++$, strong staining). The proportion and intensity scores were then summed to obtain the final immunostaining score $(A+B)$. Additionally, final scores of $0-3$ or 4-6 were divided into low or high expression, respectively.

Cell culture and cell counting kit-8 (CCK-8) detection of cell growth rate. The peptide (MTP-NRP1) targeted to the NRP1 transmembrane domain was synthesized by GENEWIZ (Suzhou, China) according to the reports of Arpel et al. [15]. Cells were cultured in Dulbecco's Modified Eagle Medium (DMEM) containing $10 \%$ fetal bovine serum, at $37^{\circ} \mathrm{C}$ in a $5 \%$ $\mathrm{CO}_{2}$ incubator. To evaluate cell proliferation, the cells were seeded in 96-well plates and grown to 50-70\% confluence. After treatment with MTP-NRP1 or PTX or the combination at a constant ratio for $24 \mathrm{~h}, 10 \mu \mathrm{l}$ of CCK- 8 reagent (Beyotime, China) was added to each well, followed by incubation for $2 \mathrm{~h}$. The absorbance at $450 \mathrm{~nm}$ was then measured using a microplate reader. A combination index (CI) was performed using data obtained from MTT assay with CompuSyn software.

Flow cytometry. Flow cytometry was performed to evaluate cell apoptosis. TNBC cells were cultured in the 6-well plate and treated with MTP-NRP1. 24 hours later, cells were harvested and prepared into single-cell suspension in PBS. Annexin V-FITC and PI solution were added into the cell suspensions following the manufacturer's instructions.

Wound healing. To assess the effect of combination treatment on cell migration, wound healing assays were performed using a cell culture insert (No. 80209; Ibidi, Munich, Germany). In brief, TNBC cells were seeded at a density of $2 \times 10^{4}$ cells into a $3.5 \mathrm{~cm}$ Petri dish containing an insert and grown overnight. The cells were then washed with PBS, the inserts were removed, and the cells were allowed to continue to grow for another $24 \mathrm{~h}$. Representative images for each group were photographed at 0 and $24 \mathrm{~h}$.

Western blot. Cells were lysed using RIPA lysis buffer at $4^{\circ} \mathrm{C}$ for $30 \mathrm{~min}$. Total protein concentrations were quantified using a colorimetric detection assay (BCA Protein Assay; Pierce, Rockford, IL, USA). An equal amount of protein was separated by $10 \%$ polyacrylamide gel electrophoresis and transferred to a nitrocellulose membrane. The membrane was blocked with 5\% skim milk and incubated with primary antibodies against PARP, caspase 3 , and $\beta$-actin (Beyotime Biotechnology, China) overnight at $4{ }^{\circ} \mathrm{C}$ followed by thricewashing for $5 \mathrm{~min}$ each with TBST. Then, the membrane was incubated with secondary antibody for $1 \mathrm{~h}$ at room temperature and thrice washed. Blotted protein samples were then detected using an enhanced chemiluminescence system (Pierce).

Statistical analyses. SPSS 16.0 software package was used for statistical analysis. NRP1 overexpression and clinicopathologic characteristics were analyzed using a chi-square test. Overall survival (OS) was estimated using the Kaplan- 
Meier method, and the log-rank test was applied to compute $\mathrm{p}$-values. The Cox proportional hazards regression model was performed toward the identification of relevant prognostic factors. A $p<0.05$ was considered statistically significant.

\section{Results}

Expression of NRP1 is positively correlated with pathologic features of advanced TNBC. In the present study, NRP1 expression was evaluated by IHC analysis in 69 TNBC patients and 60 non-TNBC patients. Whether TNBC or non-TNBC, representative immunohistochemical images of NRP1 expression showed that there was an upregulation of NRP1 expression as the pathologic stage increased (Figure 1A). At the same pTNM stage, NRP1 expression in TNBC patients was higher than in non-TNBC patients (Table 1). Our results indicate that NRP1 might be involved in $\mathrm{TNBC}$ progression.

Moreover, expression of NRP1 was positively correlated with tumor size $(p<0.001)$, axillary lymph node metastasis
A

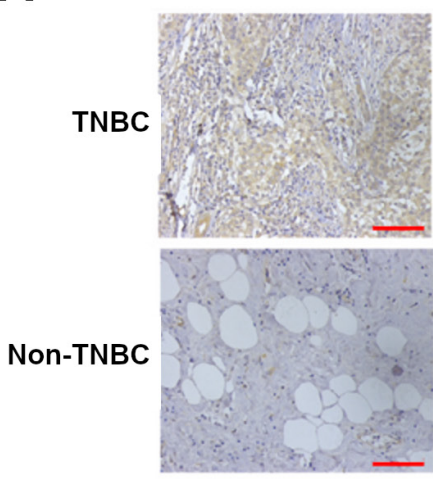

Grade II

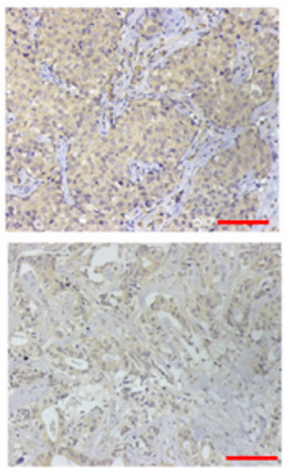

Grade III

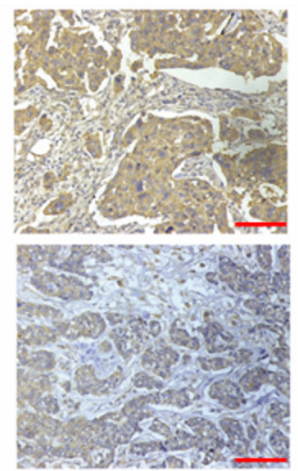

B

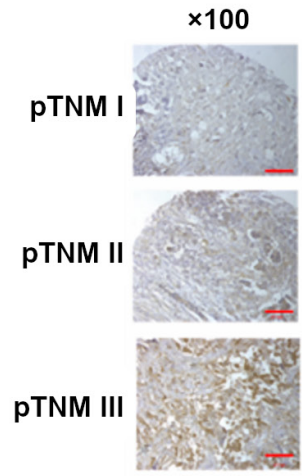

$\times 200$

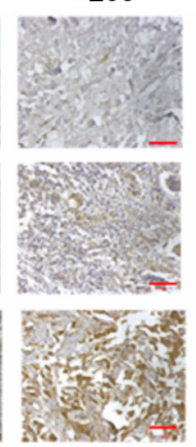

$\times 300$
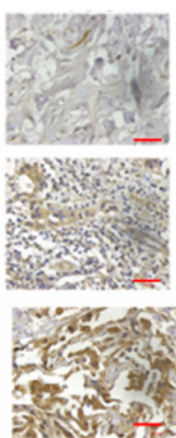

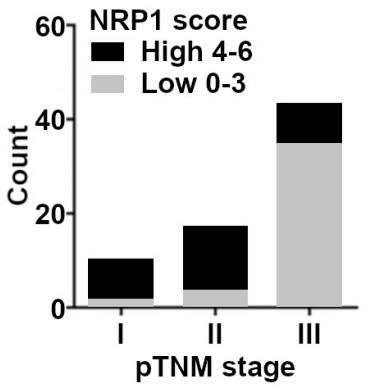

C

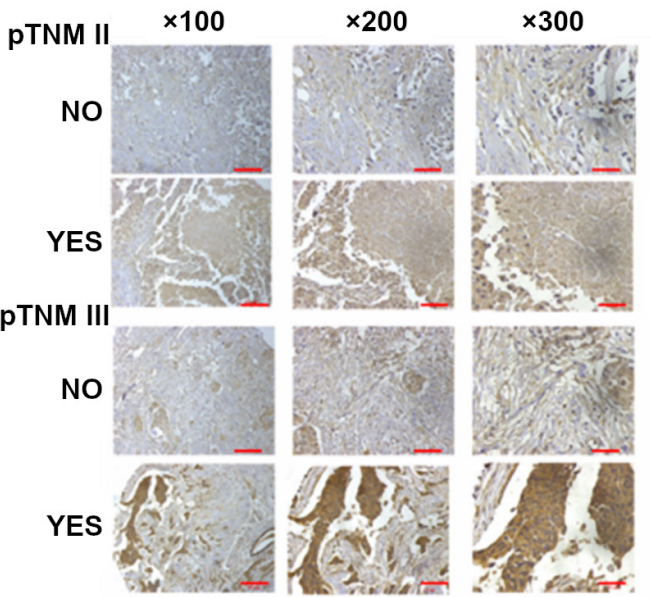

Figure 1. The expression of NRP1 was positively correlated with TNBC progression. A) The expression of NRP1 increased as the pathologic stage increased in TNBC and in non-TNBC patients. At the same pTNM stage, NRP1 expression in TNBC patients was higher than in non-TNBC patients. B) The expression of NRP1 increased significantly as the pTNM stage increased. C) The expression of NRP1 was higher in TNBC patients with axillary lymph node metastases than patients without axillary lymph node metastases. 
$(\mathrm{p}=0.001)$, degree of differentiation $(\mathrm{p}=0.001), \mathrm{pTNM}$ stage $(\mathrm{p}<0.001)$, and recurrence or distant metastasis $(\mathrm{p}=0.002)$, but not with the duration of disease (Table 2). With the increasing expression of NRP1, pTNM stage of TNBC was increased (Figure 1B). At the same time, patients with axillary lymph node metastases expressed higher NRP1 than patients

Table 1. NRP1 expression in TNBC and non-TNBC.

\begin{tabular}{lcccccc}
\hline & \multirow{6}{c}{ cases } & \multicolumn{2}{c}{ NRP1 score, $\mathbf{n}(\%)$} & \multirow{2}{*}{$\boldsymbol{\chi}^{2}$} & p-value \\
\cline { 3 - 5 } & & $\mathbf{0 - 1}$ & $\mathbf{2 - 3}$ & $\mathbf{4 - 6}$ & & \\
\hline \multirow{2}{*}{ TNBC } & \multirow{2}{*}{69} & $\begin{array}{c}10 \\
(14.493)\end{array}$ & $\begin{array}{c}16 \\
(23.188)\end{array}$ & $\begin{array}{c}43 \\
(62.319)\end{array}$ & \multirow{2}{*}{12.219} & \multirow{2}{*}{0.002} \\
\multirow{2}{*}{ non-TNBC } & 60 & $\begin{array}{c}13 \\
(21.667)\end{array}$ & $\begin{array}{c}28 \\
(46.667)\end{array}$ & $\begin{array}{c}19 \\
(31.666)\end{array}$ & & \\
\hline
\end{tabular}

${ }^{*}$ p-value was calculated by Kruskal-Wallis test

Table 2. Association of NRP1 expression with clinicopathological variables of TNBC patients.

\begin{tabular}{lcccc}
\hline \multirow{2}{*}{ Pathological features } & \multirow{2}{*}{ Cases } & \multicolumn{2}{c}{ NRP1 expression (\%) } & \multirow{2}{*}{ p-value } \\
\cline { 4 - 5 } & & Low & High & \\
\hline Age, years & 42 & $13(31.0)$ & $29(69.0)$ & \\
$\quad \leq 50$ & 27 & $13(48.1)$ & $14(51.9)$ & \\
$\quad>50$ & & & & $<0.001$ \\
Tumor sizes & 28 & $20(71.4)$ & $8(28.6)$ & \\
$\quad$ T1-T2 & 41 & $6(14.6)$ & $35(85.4)$ & \\
$\quad$ T3-T4 & & & & 0.001 \\
Axillary lymph node metastasis & 25 & $16(64.0)$ & $9(36.0)$ & \\
$\quad$ Negative & 44 & $10(22.7)$ & $34(77.3)$ & \\
$\quad$ Positive & & & & 0.001 \\
Degree of differentiation & 27 & $17(63.0)$ & $10(37.0)$ & \\
$\quad$ Good/Moderate & 42 & $9(21.4)$ & $33(78.6)$ & \\
$\quad$ Poor & & & & $<0.001$ \\
pTNM stage & 26 & $18(69.2)$ & $8(30.8)$ & \\
$\quad$ I-II & 43 & $8(18.6)$ & $35(81.4)$ & \\
$\quad$ III & & & & 0.002 \\
Recurrence/Metastasis & 22 & $14(63.6)$ & $8(36.4)$ & \\
$\quad$ No & 47 & $12(25.5)$ & $35(74.5)$ & \\
$\quad$ Yes & & & & \\
\hline
\end{tabular}

${ }^{*}$ p-value was calculated by Chi square test

Table 3. Univariate and multivariate analysis for OS in TNBC patients.

\begin{tabular}{lccccc}
\hline \multirow{2}{*}{ Variables } & $\begin{array}{c}\text { Univariate } \\
\text { analysis }\end{array}$ & & \multicolumn{3}{c}{ Multivariate analysis } \\
\cline { 2 - 3 } \cline { 5 - 6 } & p-value $^{\mathbf{a}}$ & & HR & $\mathbf{9 5 \%}$ CI & p-value \\
\hline NRP1 expression & 0.009 & & 3.175 & $3.175-1.361$ & 0.007 \\
Age, years & 0.994 & & 1.131 & $0.528-2.424$ & 0.751 \\
Tumor sizes & $<0.001$ & & 1.019 & $1.007-1.031$ & 0.002 \\
Axillary lymph node & $<0.001$ & & 3.573 & $2.033-6.282$ & $<0.001$ \\
metastasis & 0.001 & & 3.175 & $1.361-7.403$ & 0.007 \\
Degree of differentiation & $<0.001$ & 3.058 & $1.217-7.686$ & 0.017 \\
\hline
\end{tabular}

${ }^{\mathrm{a}}$ calculated by Log-rank analysis; ${ }^{\mathrm{b}}$ calculated by Multivariate Cox regression analysis; 95\% CI: confidence interval without axillary lymph node metastases (Figure 1C). The results showed that the expression of NRP1 in TNBC was gradually upregulated with an advancing histological grade.

High expression of NRP1 indicates a worse prognosis of TNBC patients. To determine whether or not the level of NRP1 expression is associated with the prognosis of TNBC patients, we analyzed 69 TNBC patients who had 60 months of follow-up evaluations. It was previously shown that during 3 years of follow-up evaluations, $23.08 \%$ of patients in the low-NRP1 group had tumor-related deaths compared with $58.14 \%$ of patients in the high-expression group. Additionally, the OS was shorter $(\mathrm{p}=0.009$, Figure $2 \mathrm{~A})$ in patients with high levels of NRP1. To further assess the prognostic value of NRP1, we performed univariate and multivariate analyses based on standard clinical characteristics. TNBC patients with a shorter cancer-specific OS had a relatively high expression of NRP1. Thus, NRP1 may emerge as an independent prognostic factor for the OS (Table 3, $\mathrm{p}<0.05$ ) in patients with TNBC. Survival analysis was also performed in TNBC patients based on tumor size, degree of differentiation, axillary lymph node metastasis, and pTNM stage. Taken together, the results indicated that high expression of NRP1 led to a worse prognosis in TNBC patients (Figures 2B-2E).

MTP-NRP1 and PTX synergistically inhibited proliferation, migration, and potentiated the apoptotic effect of PTX on TNBC cells. The only therapy regimen approved for TNBC patients following surgery is the chemotherapy (PTX); however, PTX can cause side effects. Hence, we investigated the synergistic inhibitory effect of NRP1 blockage and PTX on several TNBC cell lines. Because the transmembrane domains in the membrane receptors play a key role in cell signaling transduction, we used MTP-NRP1 to block the signaling cascade of NRP1 and found that MTP-NRP1 significantly inhibited cell proliferation in a dose-dependent manner, which was consistent with a previous study [15]; similar results were found in PTX-treated cells (Figures $3 \mathrm{~A}, 3 \mathrm{~B})$. In the combined treated group, growth inhibition was significantly enhanced in all TNBC cell lines compared to PTX treatment alone. The CI values for combination treatment were $<1$, suggesting there was synergism in the combined treatment group and MTP-NRP1 sensitized TNBC cells to PTX (Figure 3C).

In addition, to determine whether or not MTP-NRP1 plus PTX synergistically inhibited TNBC cell migration, a wound healing assay was performed. As shown in Figure 3D, cell migration was inhibited more in the combination treatment groups than in the groups treated with a single drug. Synergism between MTP-NRP1 and PTX in TNBC cell growth and migration suggested that apoptosis might also be enhanced with combined treatment. To test this hypothesis, the apoptotic effect of MTP-NRP1 alone and in combination with PTX on TNBC cells was evaluated through assessing cells for the proteolytic cleavage of PARP and caspase 3. As shown in Figure 3E, compared to treatment alone, a significantly enhanced cleavage level of PARP and caspase 3 was 
A

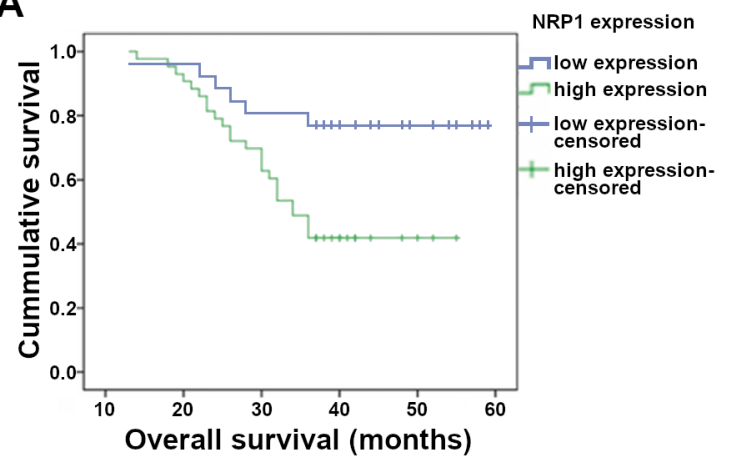

C

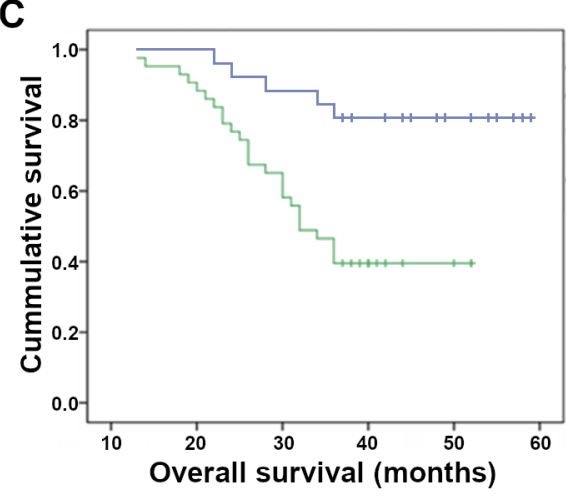

E

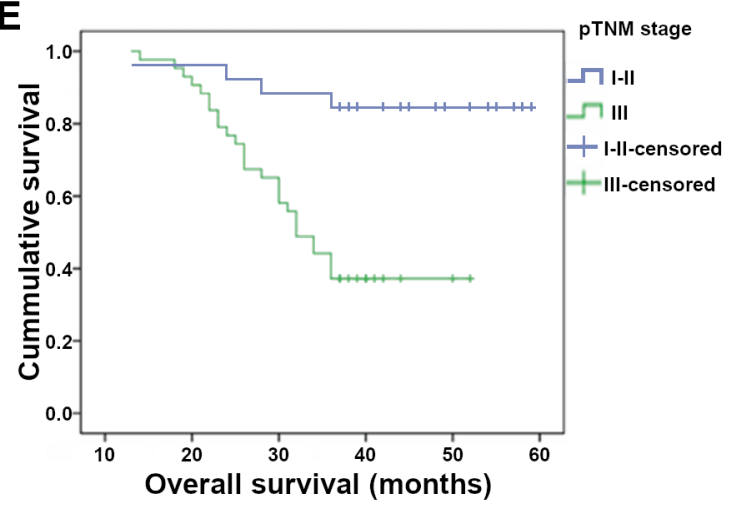

B

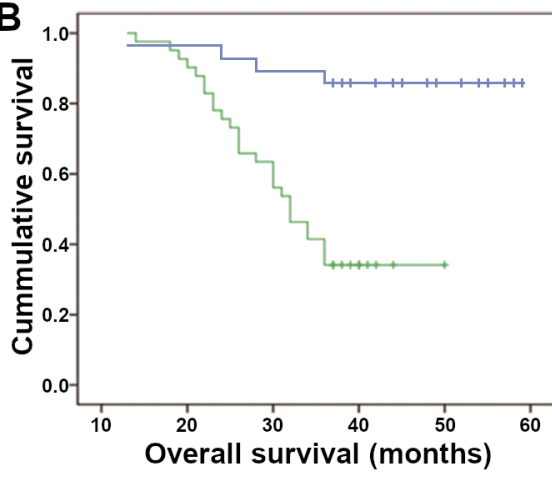

Tumor sizes

ПT1-2

$\neg$ T3-4

-T1-2 -censored

+ T3-4 -censored
D

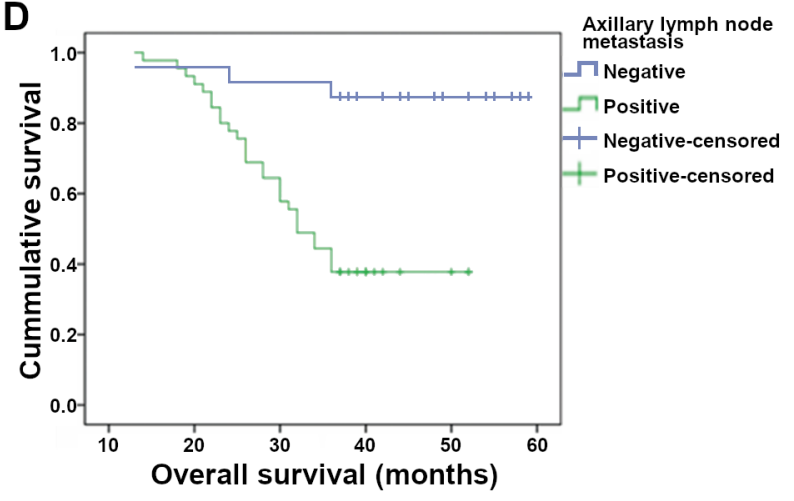

Figure 2. The relationship between the expression of NRP1 and clinical pathology in TNBC patients. Relationship between NRP1 expression (A), tumor size (B), degree of differentiation (C), axillary lymph node metastasis (D), and pTNM stage (E) with 3-year overall survival in TNBC patients.

observed in the combined treatment group. We speculated that TNBC cell proliferation inhibition by NRP1 blocker might be partially through the apoptosis induction.

\section{Discussion}

It is proposed that TNBC with a relatively poor prognosis represents a heterogeneous breast cancer subtype, which has been repeatedly reported in recent years. Patients with TNBC are characterized by a shorter OS and an early peak of distant recurrences 3 years after diagnosis $[16,17]$. TNBC, as a disease with a poor prognosis, is associated with a higher risk of local and distant relapses, which frequently occur as visceral and/or brain metastases $[18,19]$.
Angiogenesis is a characteristic biological behavior in tumors and is essential for tumor growth and metastasis. VEGF, with complex biological functions and as the most potent angiogenic factor, can induce endothelial cell proliferation, migration, and extracellular matrix remodeling, promote the capillary formation and the increase of vascular permeability. Of note, NRP1 binds members of VEGF to mediate angiogenesis $[20,21]$. Blockade of NRP1 activity represents a novel anti-angiogenic strategy because it not only inhibits angiogenesis but also vascular remodeling. Human monoclonal antibody targets the VEGF binding domain of NRP1 was the focus of a phase I clinical trial study [22].

In this study, we have demonstrated that NRP1 was highly expressed in the majority of TNBC tissue samples compared 

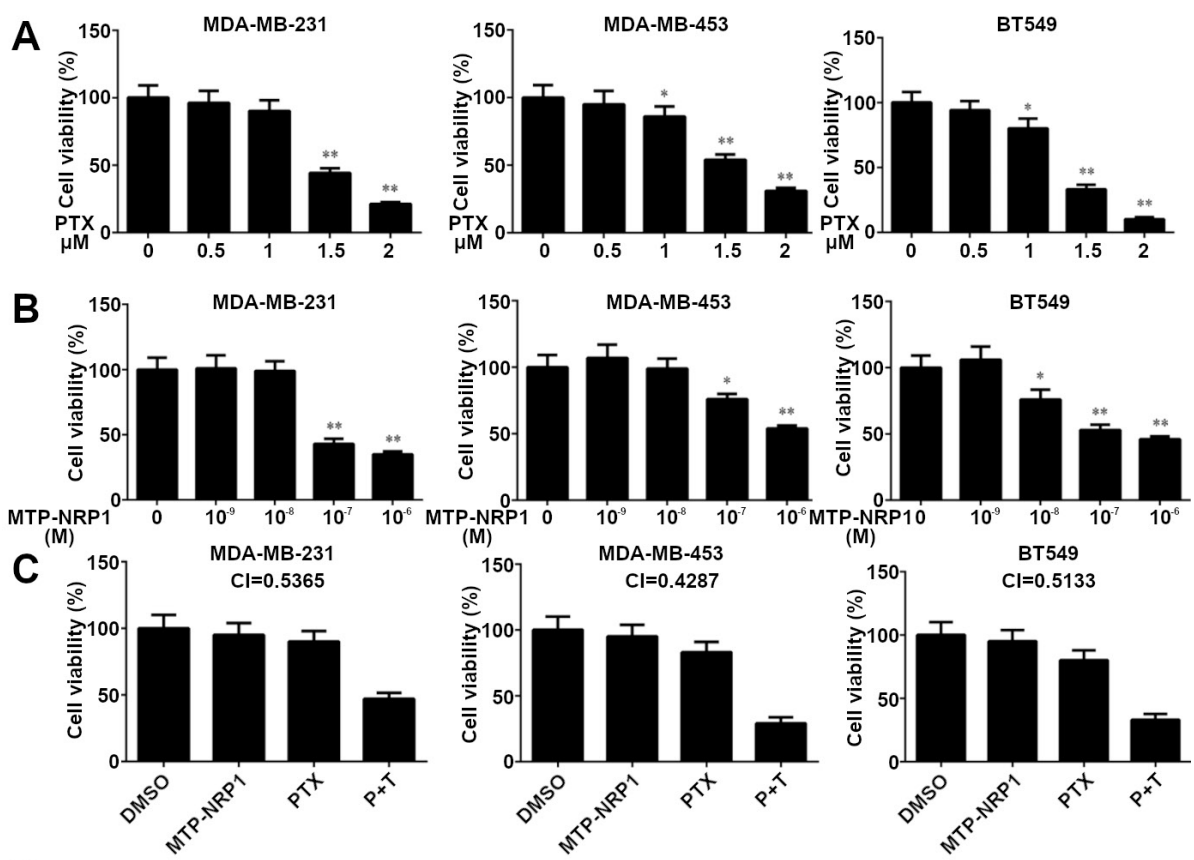

D

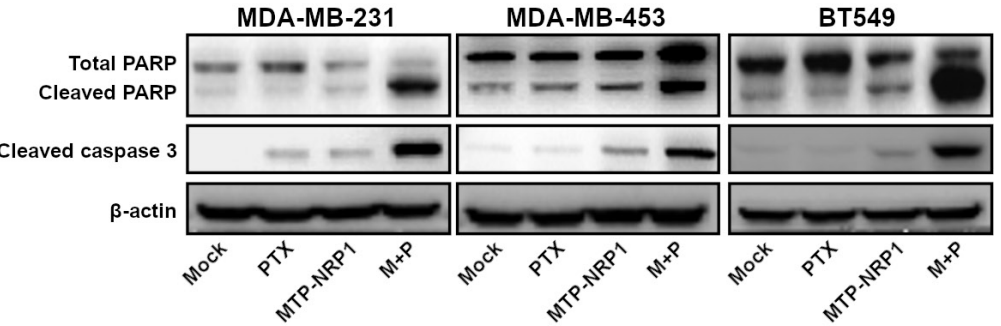

$\mathbf{E}$
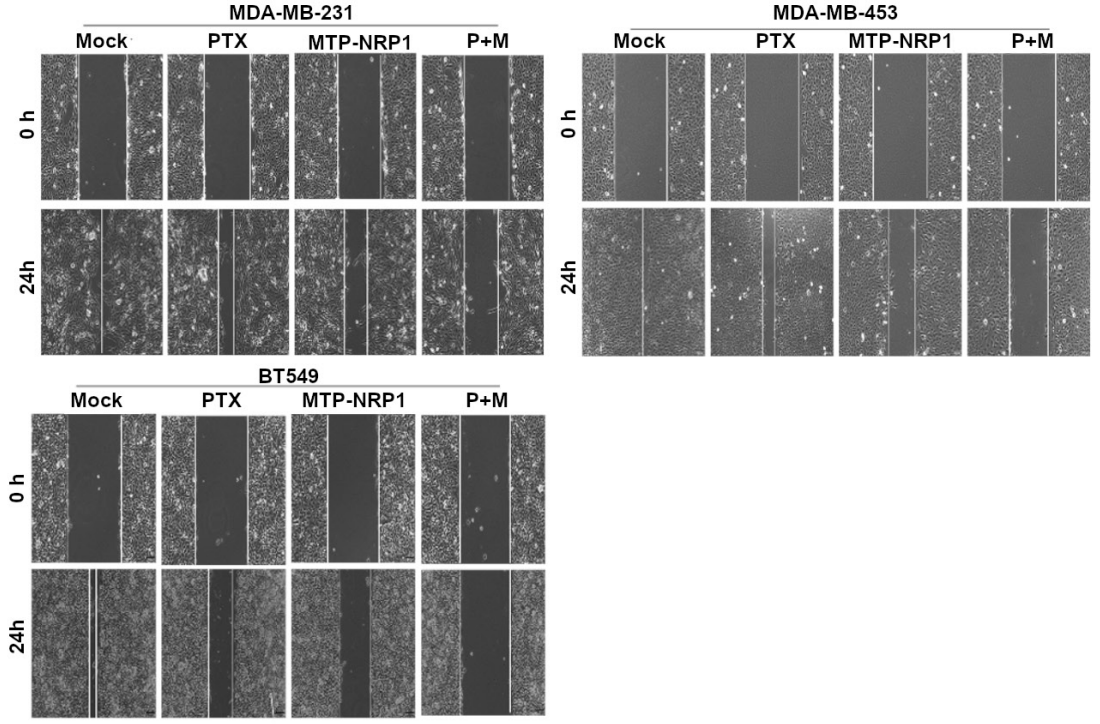

Figure 3. The combination of MTP-NRP1 and PTX inhibited TNBC cell proliferation, migration, and induced apoptosis of TNBC cells better than either drug alone. A, B) TNBC cells $\left(1 \times 10^{4}\right)$ were plated in 96-well plates and treated with MTP-NRP1 or PTX. Cell proliferation was determined at the indicated time points using an MTT assay. The results were normalized to the control group. C) Cells were treated with MTP-NRP1 ( $\left.1 \times 10^{-8} \mathrm{M}\right)$ and/or PTX $(1 \mu \mathrm{M})$, and CI values were calculated using CompuSyn software. D) TNBC cells were treated with MTP-NRP1, PTX, or the combination. Cells were allowed to migrate into the scratched area for $24 \mathrm{~h}$, and pictures were obtained with $\times 100$ magnification. E) TNBC cells were seeded in 6-well plates, and cells were treated with MTP-NRP1, PTX, and the combination. Twenty-four hours later, PARP and caspase 3 cleavage was detected by western blot. ${ }^{*} \mathrm{p}<0.05,{ }^{* *} \mathrm{p}<0.01$ 
with non-TNBC tissues. In addition, high expression of NRP1 was correlated with tumor invasion and metastasis and shortened OS in patients with TNBC. Moreover, we found that the expression of NRP1 in TNBC was gradually upregulated as the pTNM stage increased. In addition, we also demonstrated for the first time that NRP1 was more highly expressed in TNBC patients than non-TNBC patients at the same stage. Herein, we analyzed the valuable function of NRP1 in predicting prognosis in TNBC patients, and NRP1 expression can act as an independent prognostic factor for TNBC patients.

Recently, NRP1 has been reported as a multifunctional - involved in tumor initiation, growth, metastasis, and immunity [23]. Using RNA interference to knock down the RNP1 expression can inhibit the breast cancer cells viability, proliferation, and induce cell apoptosis [13, 24]; however, RNA interference can only be tested in vitro. As the transmembrane domains in the membrane receptors play a key role in the cell signaling transduction, Arpel et al. synthesized a peptide targeting the transmembrane domains of NRP1 that blocks the signaling cascade and significantly blocks cell proliferation and migration [15]. And here we demonstrated that NRP1 might enhance the curative effects and reduce the toxicity of chemotherapeutic drugs.

In summary, we demonstrated that NRP1 was overexpressed in TNBC. Overexpression of NRP1 was associated with poor prognosis in TNBC patients and was an independent prognostic factor for TNBC. In addition, NRP1 may be a potential prognostic biomarker for TNBC patients and may provide novel therapeutic targets for the future treatment of TNBC patients.

Acknowledgments: This work was supported by the Six Talent Peaks Project in Jiangsu Province (2014-wsw-040).

\section{References}

[1] SIEGEL RL, MILLER KD, JEMAL A. Cancer Statistics, 2017. CA Cancer J Clin 2017; 67: 7-30. https://doi.org/10.3322/ caac. 21387

[2] BRENTON JD, CAREY LA, AHMED AA, CALDAS C. Molecular classification and molecular forecasting of breast cancer: ready for clinical application? J Clin Oncol 2005; 23: 7350-7360. https://doi.org/10.1200/JCO.2005.03.3845

[3] HURVITZ S, MEAD M. Triple-negative breast cancer: advancements in characterization and treatment approach. Curr Opin Obstet Gynecol 2016; 28: 59-69. https://doi. org/10.1097/GCO.0000000000000239

[4] DENT R, HANNA WM, TRUDEAU M, RAWLINSON E, SUN P et al. Pattern of metastatic spread in triple-negative breast cancer. Breast Cancer Res Treat 2009; 115: 423-428. https://doi.org/10.1007/s10549-008-0086-2

[5] NAROD SA, DENT RA, FOULKES WD. CCR 20th Anniversary Commentary: Triple-Negative Breast Cancer in 2015-Still in the Ballpark. Clin Cancer Res 2015; 21: 38133814. https://doi.org/10.1158/1078-0432.CCR-14-3122
[6] CHEANG MC, VODUC D, BAJDIK C, LEUNG S, MCKINNEY $S$ et al. Basal-like breast cancer defined by five biomarkers has superior prognostic value than triple-negative phenotype. Clin Cancer Res 2008; 14: 1368-1376. https:// doi.org/10.1158/1078-0432.CCR-07-1658

[7] NEWMAN LA, REIS-FILHO JS, MORROW M, CAREY LA, KING TA. The 2014 Society of Surgical Oncology Susan G. Komen for the Cure Symposium: triple-negative breast cancer. Ann Surg Oncol 2015; 22: 874-882. https://doi. org/10.1245/s10434-014-4279-0

[8] SATODA M, TAKAGI S, OHTA K, HIRATA T, FUJISAWA H. Differential expression of two cell surface proteins, neuropilin and plexin, in Xenopus olfactory axon subclasses. J Neurosci 1995; 15: 942-955. https://doi.org/10.1523/JNEUROSCI.15-01-00942.1995

[9] GUTTMANN-RAVIV N, KESSLER O, SHRAGA-HELED $\mathrm{N}$, LANGE T, HERZOG $\mathrm{Y}$ et al. The neuropilins and their role in tumorigenesis and tumor progression. Cancer Lett 2006; 231: 1-11. https://doi.org/10.1016/j.canlet.2004.12.047

[10] KARJALAINEN K, JAALOUK DE, BUESO-RAMOS CE, ZURITA AJ, KUNIYASU A et al. Targeting neuropilin-1 in human leukemia and lymphoma. Blood 2011; 117: 920-927. https://doi.org/10.1182/blood-2010-05-282921

[11] PAN Q, CHANTHERY Y, LIANG WC, STAWICKI S, MAK $\mathrm{J}$ et al. Blocking neuropilin-1 function has an additive effect with anti-VEGF to inhibit tumor growth. Cancer Cell 2007; 11: 53-67. https://doi.org/10.1016/j.ccr.2006.10.018

[12] CHRISTENSEN C, AMBARTSUMIAN N, GILESTRO G, THOMSEN B, COMOGLIO P et al. Proteolytic processing converts the repelling signal Sema3E into an inducer of invasive growth and lung metastasis. Cancer Res 2005; 65: 61676177. https://doi.org/10.1158/0008-5472.CAN-04-4309

[13] ZHANG L, WANG H, LI C, ZHAO Y, WU L et al. VEGFA/Neuropilin 1 Pathway Confers Cancer Stemness via Activating Wnt/beta-Catenin Axis in Breast Cancer Cells. Cell Physiol Biochem 2017; 44: 1251-1262. https://doi. org/10.1159/000485455

[14] HAN Z, JIANG G, ZHANG Y, XU J, CHEN C et al. Effects of RNA interference-mediated NRP-1 silencing on the proliferation and apoptosis of breast cancer cells. Mol Med Rep 2015; 12: 513-519. https://doi.org/10.3892/mmr.2015.3405

[15] ARPEL A, GAMPER C, SPENLE C, FERNANDEZ A, JACOB L et al. Inhibition of primary breast tumor growth and metastasis using a neuropilin-1 transmembrane domain interfering peptide. Oncotarget 2016; 7: 54723-54732. https:// doi.org/10.18632/oncotarget.10101

[16] COLLIGNON J, LOUSBERG L, SCHROEDER H, JERUSALEM G. Triple-negative breast cancer: treatment challenges and solutions. Breast Cancer (Dove Med Press) 2016; 8: 93107. https://doi.org/10.2147/BCTT.S69488

[17] DENT R, TRUDEAU M, PRITCHARD KI, HANNA WM, KAHN HK et al. Triple-negative breast cancer: clinical features and patterns of recurrence. Clin Cancer Res 2007; 13: 4429-4434. https://doi.org/10.1158/1078-0432.CCR-06-3045

[18] IRVIN WJ JR., CAREY LA. What is triple-negative breast cancer? Eur J Cancer 2008; 44: 2799-805. https://doi. org/10.1016/j.ejca.2008.09.034 
[19] LIN NU, CLAUS E, SOHL J, RAZZAK AR, ARNAOUT A et al. Sites of distant recurrence and clinical outcomes in patients with metastatic triple-negative breast cancer: high incidence of central nervous system metastases. Cancer 2008; 113: 2638-2645. https://doi.org/10.1002/cncr.23930

[20] MAMLUK R, GECHTMAN Z, KUTCHER ME, GASIUNAS N, GALLAGHER J et al. Neuropilin-1 binds vascular endothelial growth factor 165, placenta growth factor-2, and heparin via its b1b2 domain. J Biol Chem 2002; 277: 24818 24825. https://doi.org/10.1074/jbc.M200730200

[21] RIZZOLIO S, RABINOWICZ N, RAINERO E, LANZETTI L, SERINI G et al. Neuropilin-1-dependent regulation of EGF-receptor signaling. Cancer Res 2012; 72: 5801-5811. https://doi.org/10.1158/0008-5472.CAN-12-0995
[22] WEEKES CD, BEERAM M, TOLCHER AW, PAPADOPOULOS KP, GORE L et al. A phase I study of the human monoclonal anti-NRP1 antibody MNRP1685A in patients with advanced solid tumors. Invest New Drugs 2014; 32: 653-660. https://doi.org/10.1007/s10637-014-0071-z

[23] PRUD'HOMME GJ, GLINKA Y. Neuropilins are multifunctional coreceptors involved in tumor initiation, growth, metastasis and immunity. Oncotarget 2012; 3: 921-939. https:// doi.org/10.18632/oncotarget.626

[24] ZHANG L, CHEN Y, WANG H, ZHENG X, LI C et al. miR-376a inhibits breast cancer cell progression by targeting neuropilin-1 NR. Onco Targets Ther 2018; 11: 5293-5302. https://doi.org/10.2147/OTT.S173416 